\title{
Comment on Liu et al.: Meta-analysis of randomised controlled trials comparing unipolar with bipolar hemiarthroplasty for displaced femoral-neck fractures
}

\author{
Yan Wang $•$ Ning Zhu $\cdot$ Qingzhuang Chen $\cdot$ Yong Wang
}

Received: 8 July 2014 / Accepted: 13 July 2014 / Published online: 12 August 2014

(C) SICOT aisbl 2014

\section{Dear Editor,}

We read with great interest the recent paper by Liu et al. "Metaanalysis of randomised controlled trials comparing unipolar with bipolar hemiarthroplasty for displaced femoral-neck fractures" published online in International Orthopaedics [1]. They reached an important conclusion that unipolar and bipolar hemiarthroplasty achieved similar clinical outcomes in patients with displaced femoral neck fractures. It is an interesting study. Nevertheless, we have several worthwhile queries which we would like to communicate with the authors.

1. Four electronic databases (PubMed, Embase, Cochrane Central Register of Controlled Trials and Web of Science) were systematically searched by the authors. The small number of acquired papers would be an important limitation of this meta-analysis. We hope that more electronic databases can be systematically searched.

2. Publication language was limited to English in this metaanalysis. Therefore, the authors should mention the potential language bias in the limitations of their meta-analysis.

3. The inclusion criteria were not enough and the exclusion criteria were not mentioned in this meta-analysis. We suggest that strict inclusion and exclusion criteria should be described in detail.

4. The authors have clearly mentioned that "Two authors independently appraised the quality of the included studies according to the Cochrane Collaboration guidelines" in the methods. However, there were no detailed scores

Y. Wang $\cdot$ Q. Chen $\cdot$ Y. Wang $(\triangle)$

Department of Pharmacy, Zhujiang Hospital, Southern Medical

University, No.253, Industrial Road, Haizhu District, Guangzhou,

China

e-mail: wy6507364@163.com

N. Zhu

Department of Pharmacy, The Third People's Hospital of Nanhai, Foshan, China for each trial. We suggest that detailed scores for each study should be provided.

5. A series of meta-analysis results were showed in the results. We consider that the authors should present forest plots for all the results.

6. The authors never mention the potential importance of publication bias in the limitations of their meta-analysis. We suggest that the publication bias needs to be assessed by visual examination of a funnel plot and statistical tests (e.g. Egger's linear regression test or Begg's rank correlation test). Therefore, publication bias may be present, distorting the meta-analysis.

7. The authors used an inverse variance (IV) random effects model to pool the data in this review. In our opinion, studies should be combined by using the DerSimonian and Laird random effects model, which considers both within- and between-study variations [2].

In conclusion, the results of the meta-analysis by Liu et al. should be interpreted with caution. To reach a definitive conclusion, more high-quality studies with larger sample sizes are still needed. And we believe that our remarks will contribute to more accurate elaboration of the results presented by Liu et al.

\author{
Acknowledgments None. \\ Conflict of interest The authors declare that they have no conflict of interest.
}

\section{References}

1. Liu Y, Tao X, Wang P et al (2014) Meta-analysis of randomised controlled trials comparing unipolar with bipolar hemiarthroplasty for displaced femoral-neck fractures. Int Orthop. doi:10.1007/s00264-014-2355-5

2. DerSimonian R, Laird N (1986) Meta-analysis in clinical trials. Control Clin Trials 7(3):177-188 